\title{
Numerical Simulation of Probe Measurements in Non-equilibrium Plasma
}

\author{
Jeremiah J. Boerner ${ }^{*}$ and Iain D. Boyd ${ }^{\dagger}$ \\ Department of Aerospace Engineering, University of Michigan, Ann Arbor, MI, 48109
}

\begin{abstract}
This study investigates the axisymmetric plasma flow field near a fixed-potential surface, representing the conditions near a Faraday cup. A hybrid computational code simulates electrons with a fluid model using the Boltzmann relation, and heavy particles with a Particle In Cell method. The planar Bohm sheath solution is recovered accurately for plasma conditions $n_{i}=1.1 \times 10^{14} \mathrm{~m}^{-3}, T_{i}=300 \mathrm{~K}, T_{e}=1 \mathrm{eV}$. Conditions representative of a lowpower Hall thruster plume $\left(n_{i}=1.1 \times 10^{14} \mathrm{~m}^{-3}, v_{i}=2,380 \mathrm{~m} / \mathrm{s}, T_{i}=1 \mathrm{eV}, T_{e}=1 \mathrm{eV}\right)$ also show good agreement with Bohm sheath theory. Simulated measurements of ion current on a $2 \mathrm{D}$ probe geometry are $10-20 \%$ higher than expected from elementary theory, suggesting a focusing effect of electric fields in the sheath.
\end{abstract}

\section{Nomenclature}

$\begin{array}{ll}A & =\text { probe area } \\ e & =\text { electron charge } \\ \varepsilon_{0} & =\text { permittivity of free space } \\ \Gamma_{s} & =\text { ion flux at sheath edge } \\ k_{B} & =\text { Boltzmann constant } \\ L_{D} & =\text { Debye length } \\ M & =\text { Mach number with respect to Bohm velocity } \\ m_{i} & =\text { ion mass } \\ n_{e} & =\text { electron number density } \\ n_{i} & =\text { ion number density } \\ \Phi(x) & =\text { local potential } \\ T_{e} & =\text { electron temperature } \\ T_{i} & =\text { ion temperature } \\ u(x) & =\text { ion velocity } \\ v_{B} & =\text { Bohm velocity } \\ v_{i} & =\text { ion drift velocity } \\ x & =\text { sheath coordinate }\end{array}$

\section{Introduction}

broad family of spacecraft propulsion concepts us
systems are collectively referred to as Electric Propulsi
based on the particular mechanism for generating thrust ${ }^{1}$ : e
resistance to heat a propellant before expanding through a
thrusters) apply external electric fields to accele
(magnetoplasmadynamic thrusters) apply magnetic fields to
Electric propulsion devices can achieve very high exhaus
thrusters to tens of kilometers per second for ion thrusters. Th
and outer planet missions. ${ }^{2}$ The associated specific impulse
surface) is also high, ranging from 1,000 to 4,000 seconds
${ }^{*}$ Graduate Student, (jboerner@umich.edu)
${ }^{\dagger}$ Professor, AIAA Associate Fellow (iainboyd@umich.edu) 
rockets. Therefore an EP system requires much less propellant mass than a chemical system for a fixed total impulse. Alternatively, an EP system will have a longer useful lifetime for a given propellant mass.

Ongoing development of next-generation Hall thrusters and ion engines includes life tests, performance evaluation, and spacecraft integration. An important concern in the use of EP thrusters is the possible impingement of high energy ions on spacecraft surfaces, which may cause material erosion. Measurements of plasma properties in the exhaust plume are used to evaluate the performance and characterize the conditions around an operating thruster. Common experimental diagnostics include the Faraday cup, Langmuir probe, and Retarding Potential Analyzer (RPA).$^{3-5}$ Each of these instruments is immersed in the exhaust plume and may affect the plasma condition with both physical obstruction and electrostatic sheaths. These effects make it difficult to recover undisturbed plasma conditions from the measurements.

This paper compares the results of numerical simulation of a Faraday probe with electrostatic Bohm sheath theory. The planar Bohm sheath solution is presented in a non-dimensional form, followed by profiles of plasma potential and number density for conditions found downstream of a low power Hall thruster. The computational code is described briefly, with emphasis on the geometries used in this work. Results from numerical simulation are presented for both flat plate and cylindrical probe geometries.

\section{Planar Sheath Theory}

Electrostatic probes are simple, inexpensive diagnostics that can be used to measure a number of plasma parameters. A Faraday cup is simply a current collecting surface, typically biased to repel electrons, which measures ion flux. The most basic Langmuir probe is little more than a wire inserted into a plasma. A record of collected current over a range of bias voltage allows the electron temperature, plasma potential, floating potential, and number density to be determined.

The presence of a fixed-potential surface in the plasma necessitates the formation of a sheath. The sheath structure is often divided as in Fig. 1, featuring undisturbed neutral plasma far from the surface, an approximately neutral presheath region with small potential gradient, and a non-neutral sheath within a few Debye lengths of the surface.

\section{A. Bohm Sheath Solution}

For a collisionless planar sheath with cold ions, continuity and conservation of energy for the ions can be expressed in terms of the ion density, $n_{i}$, and velocity, $u_{i}$, as

$$
\begin{gathered}
n_{i}(x) u_{i}(x)=n_{i s} u_{i s} \\
\frac{1}{2} m_{i} u_{i}^{2}(x)+e \phi(x)=\frac{1}{2} m_{i} u_{i s}^{2}
\end{gathered}
$$

where the subscript $s$ refers to values at the edge of the sheath. The sheath edge $(x=0)$ is properly defined as the point where the electron and ion number densities are equal.

The ion number density can be related to the potential alone by eliminating $u_{i}(x)$ in the preceding equations.

$$
n_{i}(x)=n_{i s}\left(1-\frac{2 e}{m u_{i s}^{2}} \phi(x)\right)^{-\frac{1}{2}}
$$

For a steady sheath, the electrons assume a Maxwellian distribution. The electron number density is then determined from the local potential through the Boltzmann relation.

$$
n_{e}(x)=n_{i s} \exp \left(\frac{e \phi(x)}{k_{B} T_{e}}\right)
$$

Poisson's equation closes the set by relating the potential to the ion and electron number densities in the sheath. 


$$
\frac{d^{2} \phi(x)}{d x^{2}}=-\frac{e}{\varepsilon_{0}}\left(n_{i}(x)-n_{e}(x)\right)
$$

The plasma is assumed to have zero potential and no electric field at the sheath edge, which provides the necessary boundary conditions.

$$
\phi(0)=0 \text { and } \frac{d \phi(0)}{d x}=0
$$

A second-order differential equation for the potential is obtained when Eqs. (3)-(4) are inserted into Eq. (5). ${ }^{6-7}$ Normalizing the potential by the electron temperature suggests the Debye length, $L_{D}$, and Bohm velocity, $v_{B}$, as appropriate length and velocity scales. The Mach number based on Bohm velocity, $M$, is the sole parameter.

$$
\begin{gathered}
\frac{d^{2} \Phi}{d z^{2}}=\left(1+\frac{2}{M^{2}} \Phi\right)^{-\frac{1}{2}}-\exp (-\Phi) \\
\text { with } \Phi(0)=0 \text { and } \frac{d \Phi(0)}{d x}=0 \\
\text { and } \Phi=-\frac{e \phi(x)}{k_{B} T_{e}}, z=\frac{x}{L_{D}}, M=\frac{u_{i s}}{v_{B}}, L_{D}=\sqrt{\frac{\varepsilon_{0} k_{B} T_{e}}{e^{2} n_{i s}}} \text {, and } v_{B}=\sqrt{\frac{k_{B} T_{e}}{m_{i}}}
\end{gathered}
$$

Equation (7) can be integrated analytically from the sheath edge to an arbitrary point $z$ to yield the form shown in Eq. (8), but a numerical technique is required for the second integration. Once the potential has been found, the ion and electron properties can be recovered using Eqs. (1)-(4).

$$
\frac{1}{2}\left(\frac{d \Phi}{d z}\right)^{2}=\exp (-\Phi)-1-M^{2}\left[\left(1+\frac{2}{M^{2}} \Phi\right)^{\frac{1}{2}}-1\right]
$$

Taking a Taylor expansion about $z=0$ yields the condition in Eq. (9) which shows that the solution is purely real only if $M^{2}>1$. That is, ions must enter the sheath edge at the Bohm velocity or faster to develop a stable sheath.

$$
\left(1-\frac{1}{M^{2}}\right) \frac{1}{2} \Phi^{2}>0
$$

Ions in the exhaust plume of an EP device are typically supersonic and this condition is easily satisfied. In other applications where the ions are slow moving or stationary, a presheath structure forms to accelerate ions up to the Bohm velocity at the sheath edge.

\section{B. Plasma Conditions and Particular Solution}

Undisturbed plasma properties are selected as representative of flow downstream of an EP thruster. In particular, we choose values from $50 \mathrm{~cm}$ downstream and $75^{\circ}$ off-axis in the exhaust plume of a Busek Co. "BHT-200" $200 \mathrm{~W}$ xenon Hall thruster. This is a region of interest since experimental measurements suggest that a significant fraction of the ion flux in this region is due to charge exchange ions; that is, low energy ions formed by collisions downstream of the thruster's acceleration region. This low-energy population may be focused by electric fields near 
the probe and lead to an over-estimate of the ion current. Calculations that rely on the measured current, such as integrated discharge current, will be skewed accordingly.

Previous numerical simulations of the BHT-200 flow field provide the values reported in Table $1 .^{8}$ Experimental Langmuir probe measurements taken by $\mathrm{Ma}$ at the same location give similar magnitude values for ion number density and electron temperature. ${ }^{9}$ In that experimental setup, the Faraday probe is biased to $-15 \mathrm{~V}$ to repel electrons.

The Bohm sheath solution is obtained for ions entering at the Bohm velocity as described in the preceding section. Figures 2-4 respectively show profiles of potential, electron number density, and ion number density in the sheath. Ion and electron number densities are nearly $10 \%$ below the undisturbed values even at 15 Debye lengths $(1.06 \mathrm{~cm})$ from the probe surface. The plasma potential at that point is non-trivial, $-0.11 \mathrm{~V}$. As will be seen in later sections, the sheath structure is compressed to about 8 Debye lengths for ions entering at the higher plume velocity.

As a note to interpreting these figures, ion flux is conserved throughout the sheath but electron flux is not. Therefore, the decrease of ion density depicted in Fig. 4 indicates an increase in velocity. In contrast, the decrease in electron density in Fig. 3 corresponds to a smaller fraction of the distribution with kinetic energy sufficient to reach the local potential.

\section{Model Description}

The computational model simulates 2D axisymmetric flow using a hybrid Particle In Cell (PIC) method. Electrons are treated with a fluid model, while ions and neutrals are simulated with a PIC model. ${ }^{10}$ The cylindrical Faraday probe geometry lends itself to a computational grid consisting of equally spaced rectangular cells.

Two models are available for simulating the electron fluid. The first is a detailed fluid model that explicitly tracks electron continuity, momentum, and energy. The second is a simplified Boltzmann model where a streamfunction is used instead of the continuity equation, and the Boltzmann relation is used in place of the momentum equation. Comparisons of preliminary results from both models show negligible differences and confirm that the electrons remain isothermal. Since the Bohm sheath solution assumes that electrons follow the Boltzmann relation, the Boltzmann model is used in the results that follow.

Ions and neutrals are simulated with the PIC module. Particle weights (the number of real atoms represented by a simulated particle) are varied in steps from the centerline to the outer edge of the domain. This limits the number of simulated particles, thereby reducing the total computation time. Cell properties are determined from a weighted average of the particles within. A self-consistent potential field is calculated from Poisson's equation using an Alternating Direction Implicit (ADI) solver. Electrostatic fields are then calculated from the gradient of the potential and applied to the particles. A Direct Simulation Monte Carlo (DSMC) routine ${ }^{11}$ is in place to handle collisions, although the plasma conditions in this study are very nearly collisionless.

In order to investigate two-dimensional (2D) effects of the probe geometry, the two computational domains in Fig. 5 are used. In both geometries the probe radius is $0.952 \mathrm{~cm}$, equal to the radius of the probe used experimentally in Ref 9. The first geometry represents a "flux tube" cylinder upstream of the collecting surface. This domain approximates an infinite plane through a specular reflection boundary condition at the outer radial edge. Results from this geometry should vary only along the flow direction, as in the Bohm sheath formulation.

The second geometry represents an immersed cylindrical probe. The entire volume of the flux tube mesh is included as a subset in front of the probe, with additional radial domain to capture behavior alongside the probe. In this case the outer radial edge has an inlet boundary condition that introduces particles with the same distribution as the upstream inlet. Radial gradients in potential and electron properties are set to zero along this edge.

Both computational grids require several additional boundary conditions. At the upstream edge is an inlet that generates the ion particles which enter the domain during a time step. The velocity of each particle is selected from a Maxwellian distribution using an acceptance/rejection method. Ion thermal temperature and drift velocity are included as inputs. Potential is set to $-15 \mathrm{~V}$ along the probe front face and side wall, consistent with the experimental probe in Ref. 9. At probe surfaces, ion particles undergo a diffuse reflection and are converted to neutral particles. In the probe geometry, particles are removed as they cross out of the domain at the downstream edge beyond the probe radius. Potential and electron property gradients normal to the edge are set to zero. 
The Bohm sheath solution provides an estimate of the required domain length at approximately 15 Debye lengths. The appropriate radial extent for the probe geometry is not so neatly provided, but is set one half-radius beyond the probe edge. Previous experience with the computational code suggests that the maximum cell spacing should be at least a factor of 15 smaller than the Debye length. Rounding in favor of conservative values, the cells are dimensioned at $4 \times 10^{-5} \mathrm{~m}$ on a side. The flux tube geometry extends 266 cells $(1.06 \mathrm{~cm})$ upstream of the probe and 238 elements $(0.952 \mathrm{~cm})$ along the probe face for a total of 63,308 cells. The probe geometry encompasses 390 cells along the axis $(1.56 \mathrm{~cm})$ and 365 cells radially $(1.46 \mathrm{~cm})$ for a total of 112,838 cells outside of the probe.

The simulation time step is selected so that the fastest ions travel less than one cell length per iteration. For the plume conditions, ions that enter at twice the thermal speed beyond the drift velocity arrive at the probe with a velocity of $6,220 \mathrm{~m} / \mathrm{s}$. Dividing the cell length by this speed and rounding down gives the time step at $5 \times 10^{-9} \mathrm{~s}$.

Each simulation is run for 20,000 iterations to reach a steady state, followed by 10,000 sampled iterations. Particle counts are typically 800,000 for the flux tube geometry and 1,800,000 for the probe geometry. When run on a $3.8 \mathrm{GHz}$ Pentium 4 system, a flux tube simulation can be completed in 6 hours and a probe simulation is more expensive at 15 hours.

\section{Results}

As a validation of the computational code, a baseline run was performed with relatively cold ions entering the domain at the Bohm velocity. Subsequent simulations increased ion temperature or inlet velocity separately to identify any departures from the Bohm sheath theory. Ultimately, the desired simulation was performed with both a high ion temperature and the full velocity found in the thruster plume.

Parameters and boundary conditions for the baseline and thruster plume simulations are reported in Table 2. Results from the intermediate simulations are not presented here, since departures from Bohm sheath theory were consistently small. One point worth noting from the intermediate simulations is that the ion temperature has relatively small effect on the final flow field. This is perhaps anticipated from a kinetic viewpoint: applying an acceleration to a distribution will drive it toward a monoenergetic state.

Table 2. Inflowing plasma properties.

\begin{tabular}{rrr} 
& Bohm inlet & Plume inlet \\
\hline$n_{i}$ & $1.1 \times 10^{14} \mathrm{~m}^{-3}$ & $1.1 \times 10^{14} \mathrm{~m}^{-3}$ \\
$T_{i}$ & $300 \mathrm{~K}$ & $1 \mathrm{eV}$ \\
$T_{e}$ & $1 \mathrm{eV}$ & $1 \mathrm{eV}$ \\
$v_{i s}$ & $855 \mathrm{~m} / \mathrm{s}$ & $2380 \mathrm{~m} / \mathrm{s}$ \\
$M$ & 1.0 & 2.78 \\
\hline
\end{tabular}

\section{A. Flux Tube Simulations}

The specular boundary condition at the outer radius of this domain produces a radially-uniform flow field as desired. Contour plots of potential and electron number density (Figs. 6-9) clearly demonstrate that the profiles are very close to one-dimensional. A small degree of scatter appears in the ion number density contours (Figs. 10-11), primarily at the radii where particle weights are increased.

Particle averages taken over the entire radial extent are compared with Bohm sheath profiles of potential (Figs. 12-13), electron density (Figs. 14-15), and ion density (Figs. 16-17). In this format the agreement between simulation and theory is seen to be very good for both inflow conditions, with agreement better than $10 \%$ throughout the domain.

\section{B. Probe Geometry Simulations}

Contour plots of the probe geometry flow field (Figs. 18-23) show definite radial variation. From centerline to outer radius of the probe, electron and number densities can climb as much as $2 \times 10^{13} \mathrm{~m}^{-3}$ while plasma potential varies 1 to $2 \mathrm{~V}$. These variations are more pronounced for the Bohm velocity inflow condition where lateral diffusion is more effective.

The outermost edge of the domain is too close to the probe, as evidenced by the significant change in curvature of the contours beyond $r=0.012 \mathrm{~m}$. Although the effect seems to be localized to the outermost 1 to $2 \mathrm{~mm}$, additional simulations on an extended domain are needed. The radial extent of the sheath is likely to be comparable or larger than the axial extent due to the lower transverse velocity.

Radial averages of the plasma properties include only the first 100 cells $(4 \mathrm{~mm})$ to exclude significant 2D effects while producing smooth ion density profiles. These profiles still show very good agreement with Bohm sheath profiles of potential (Figs. 24-25), electron density (Figs. 26-27), and ion density (Figs. 28-29). Some influence of the 2D nature appears even in this limited sample, and is most evident in the potential profile for the Bohm inlet velocity. The average potential lies below the sheath theory curve in the flux tube geometry (Fig. 12) but is pulled above it in the probe geometry (Fig. 24). 
Here the probe radius is sufficiently large to recover a region of one-dimensional sheath near the centerline. If the probe radius were smaller, edge effects would eliminate that region and the flow field would deviate from the Bohm sheath solution. Specifically, the plasma would remain near the undisturbed condition until closer to the probe. Gradients in potential and density would consequently be steeper. An accurate theoretical treatment of the sheath on small-diameter probes requires a 2D formulation.

\section{Simulated Ion Current Measurements}

One interesting application of this work is to simulate the measurement of plasma properties. An experimental Faraday probe collects ion current, corresponding to an integration of ion flux to the probe surface. An equivalent process is easily performed when processing particle movement in the preceding simulations. Ion collisions with the probe surface are recorded and weighted by the area of the impacted edge. A sum over all the weighted current bits gives the total collected ion current.

An estimate for the expected current is available from the Bohm sheath solution. Since ion flux is conserved throughout the sheath, the ion flux at the surface should be equal to that at the sheath edge. The collected current is then simply the product of ion charge, $e$, ion flux, $\Gamma_{s}$, and probe area, $A$.

$$
I=e A \Gamma_{s}=e A n_{i s} u_{i s}
$$

The anticipated total current is reported in Table 3 along with simulated measurements from both geometries. The flux tube geometry reproduces ion currents exactly as expected from 1D theory. The probe geometry, however, shows collected currents that are 10 to $20 \%$ higher than anticipated. This suggests that ions are focused onto the probe by electric fields in the sheath. In order to reconcile the elevated current measurement with conditions in the plasma, a larger "effective" probe area may be appropriate.
Table 3. Expected and simulated ion currents collected at the probe face, $10^{-6} \mathrm{~A}$.

\begin{tabular}{rrr} 
& Bohm inlet & Plume inlet \\
\hline Sheath edge current & 4.3 & 11.9 \\
Flux tube current & 4.2 & 11.9 \\
Probe current & 5.2 & 13.3 \\
\hline
\end{tabular}

\section{Conclusion}

A hybrid PIC code was used to simulate axisymmetric plasma flow near a Faraday probe. Both 1D and 2D flow fields were obtained through distinct computational domains. Two inlet conditions were used; the first condition tested the computational code against planar Bohm sheath theory and the second reproduced flow conditions representative of a low-power Hall thruster plume. The planar Bohm sheath solution was a reliable predictor for plasma potential, ion number density, and electron number density.

Prominent 2D features were identified in the probe geometry simulations. Noting the excess in simulated ion current is an encouraging first step toward the improvement of diagnostic techniques. Additional simulation will help to understand how plasma diagnostics affect the plasma, and how measured properties relate to the undisturbed values. Continuing work will extend the radial length of the probe geometry domain in order to insure gridindependent structures alongside the probe body. Based on the low transverse velocity, the length of the radial sheath is likely to be comparable to the upstream sheath length.

\section{Acknowledgments}

Support for this work is provided by the Jet Propulsion Laboratory with Lee Johnson as the primary contact. The authors also thank Tammy Ma and David Conroy for sharing their experimental data.

\section{References}

\footnotetext{
${ }^{1}$ Jahn, R. G. and Choueiri, E. Y., "Electric propulsion,” Encyclopedia of Physical Science and Technology, Vol. 5, 3rd ed., Academic Press, 2002, pp. 125-141.

${ }^{2}$ Dunning, J. and Jankovsky, R., "NASA’s Electric Propulsion Program,” AIAA-2003-4437, 39th AIAA/ASME/SAE/ASEE Joint Propulsion Conference, Huntsville, Alabama, July 20-23, 2003.

${ }^{3}$ Fife, J. M., Hargus, W. A., et al., "Spacecraft Interaction Test Results of the High Performance Hall System SPT-140," AIAA-2000-3521, 36th AIAA/ASME/SAE/ASEE Joint Propulsion Conference, Huntsville, Alabama, July 20-23, 2000.

${ }^{4}$ Hofer, R. R., Hass, J. M., Gallimore, A. D., "Ion Voltage Diagnostics in the Far-field Plume of a High Specific Impulse Hall Thruster," AIAA-2003-4556, 39th AIAA/ASME/SAE/ASEE Joint Propulsion Conference, Huntsville, Alabama, July 20-23, 2003.
} 
${ }^{5}$ King, L. B., Gallimore, A. D., Maresse, C. M., "Transport-Property Measurements in the Plume of an SPT-100 Hall Thruster,” Journal of Propulsion and Power, Vol. 14, No. 3, May-June 1998, pp. 327-335.

${ }^{6}$ Lieberman, M., Lichtenberg, A., Principles of Plasma Discharges and Materials Processing, John Wiley \& Sons, New York, NY, 1994, pp. 154-160.

${ }^{7}$ Chen, Francis, Introduction to Plasma Physics and Controlled Fusion, Vol. 1, Plenum Press, New York, NY, 1984.

${ }^{8}$ Boyd, I. D. and Yim, J. T., "Modeling of the Near Field Plume of a Hall Thruster," Journal of Applied Physics, Vol. 95, 2004, pp. 4575-4584.

${ }^{9} \mathrm{Ma}$, Tammy, "Quantification with Two-Dimensional Spatial Resolution of the Ion Flux Emitted from a $200 \mathrm{~W}$ Hall Effect Thruster,” California Institute of Technology, Pasadena, California, 2004, (unpublished).

${ }^{10}$ Birdsall, C. K. and Langdon, A. B., Plasma Physics Via Computer Simulation, Adam Hilger Press, 1991.

${ }^{11}$ Bird, G. A., Molecular Gas Dynamics and the Direct Simulation of Gas Flows, No. 42 in Oxford Engineering Science Series, Oxford University Press, 1994.
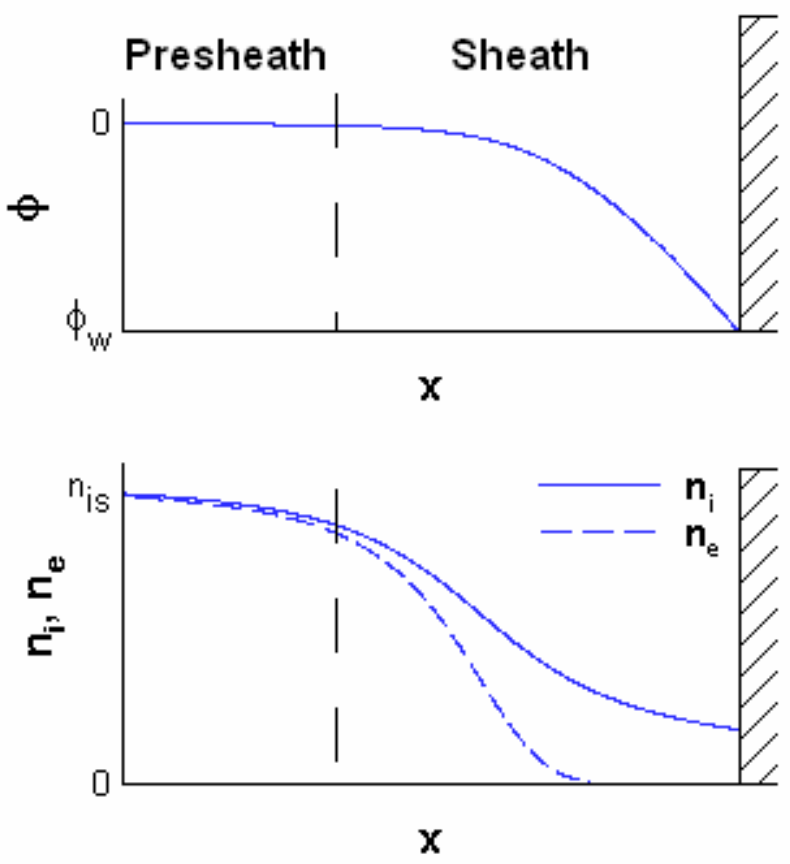

Figure 1. Schematic planar sheath structure.

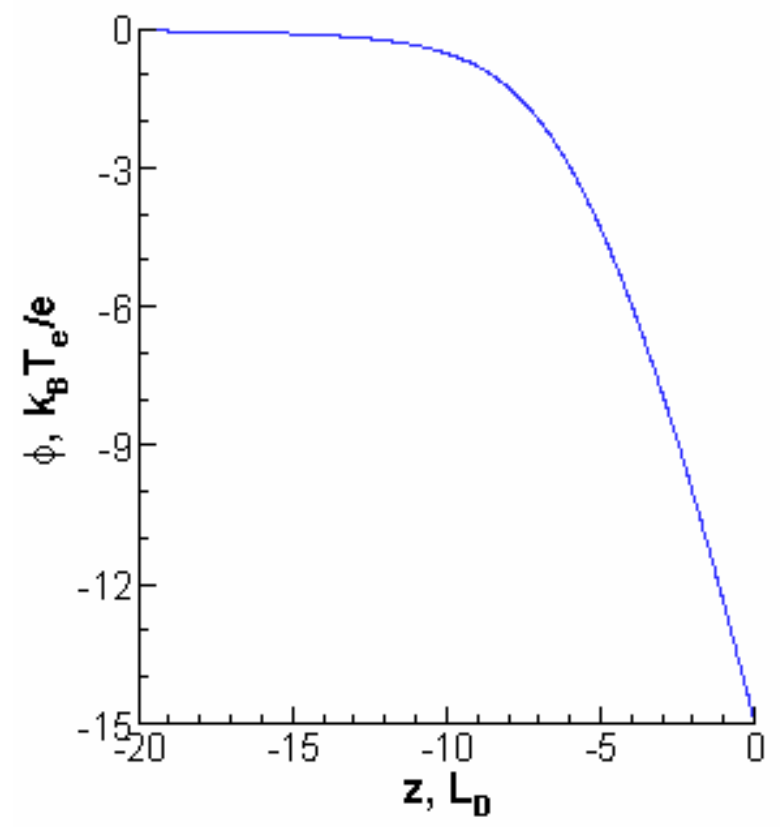

Figure 2. Bohm sheath potential profile for ions drifting at the Bohm velocity $v_{B}$ and applied surface potential $\phi_{w}=-15 \mathrm{~V}$. 


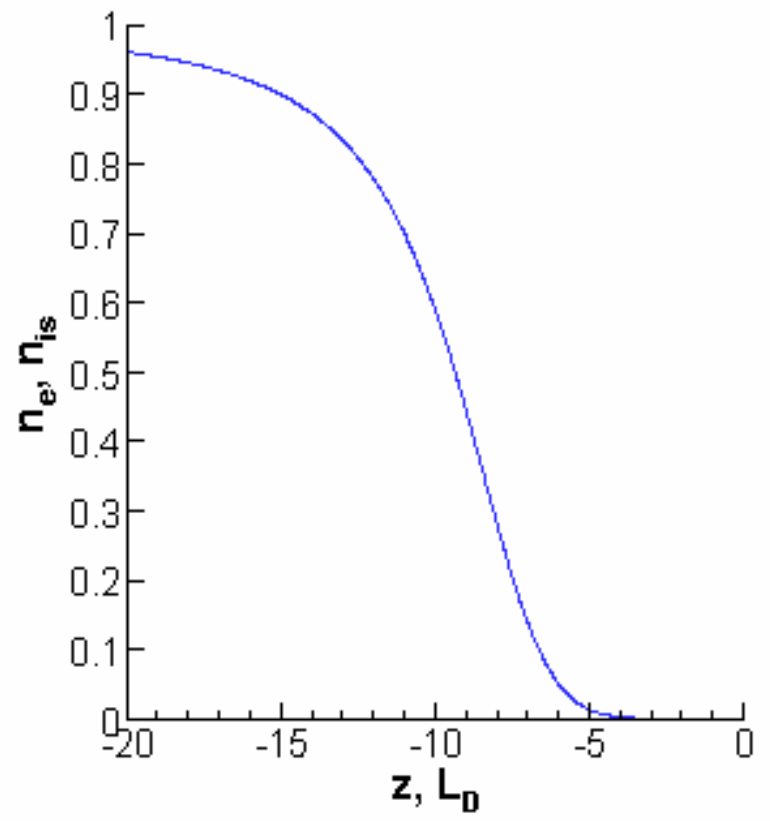

Figure 3. Bohm sheath electron number density profile for ions entering at $v_{B}$ and $\phi_{w}=-15 \mathrm{~V}$.

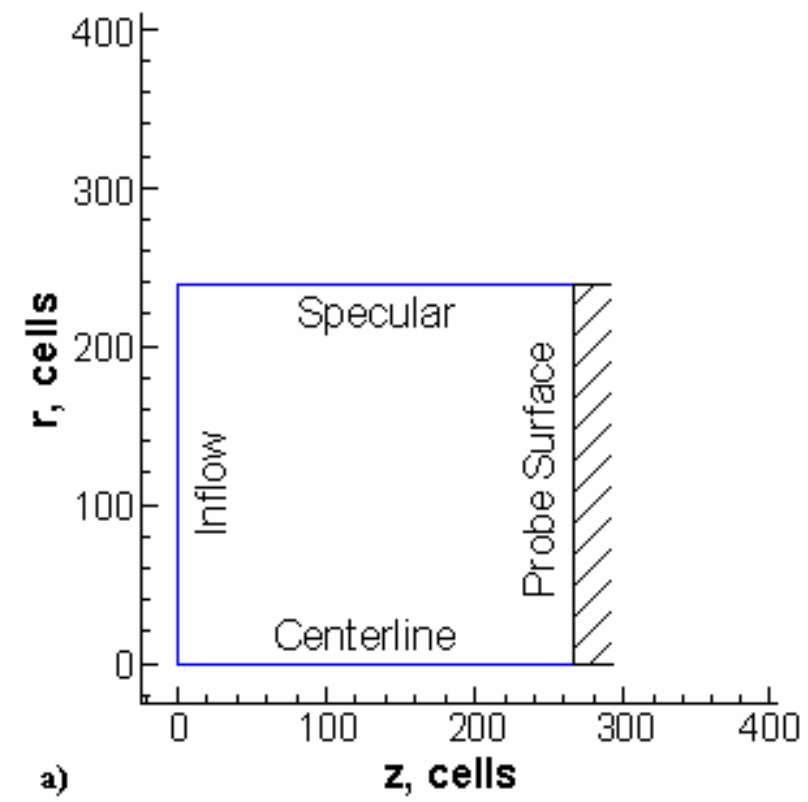

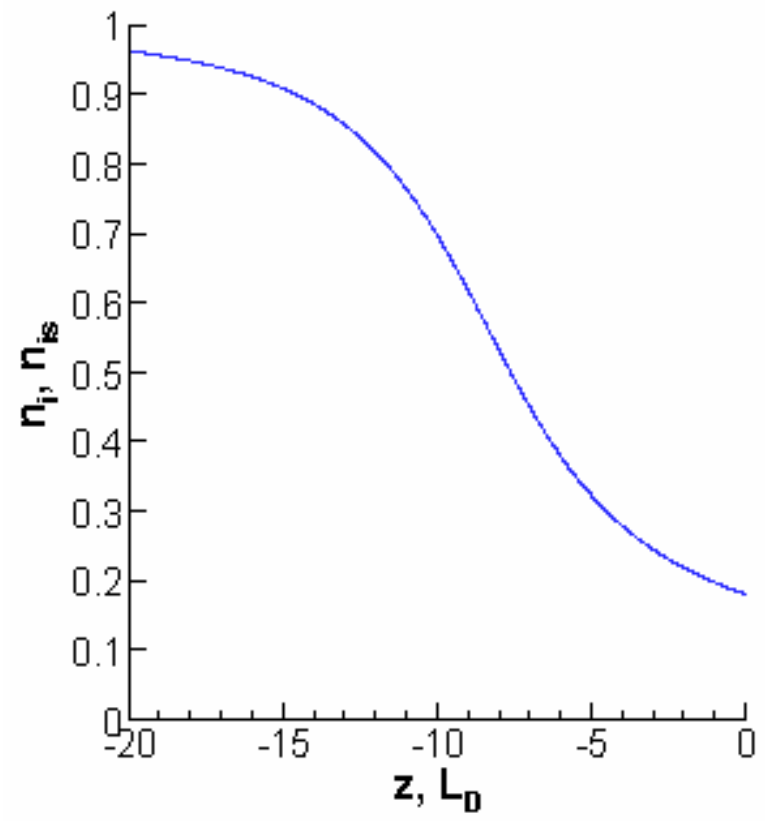

Figure 4. Bohm sheath ion number density profile for ions entering at $v_{B}$ and $\phi_{w}=-15 \mathrm{~V}$.

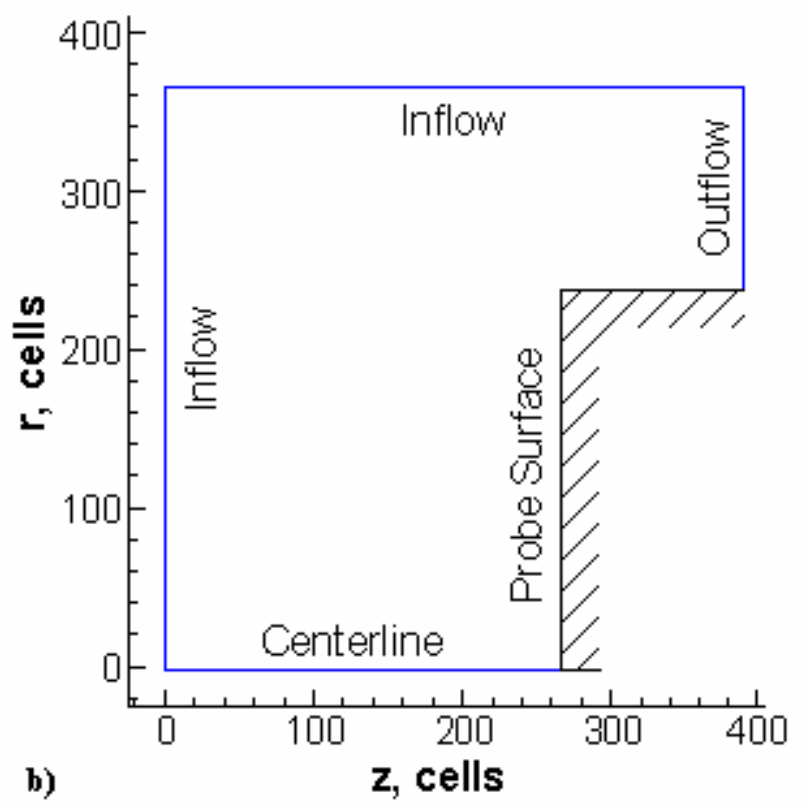

Figure 5. Computational domains used for a) flux tube simulations and b) probe geometry simulations. 


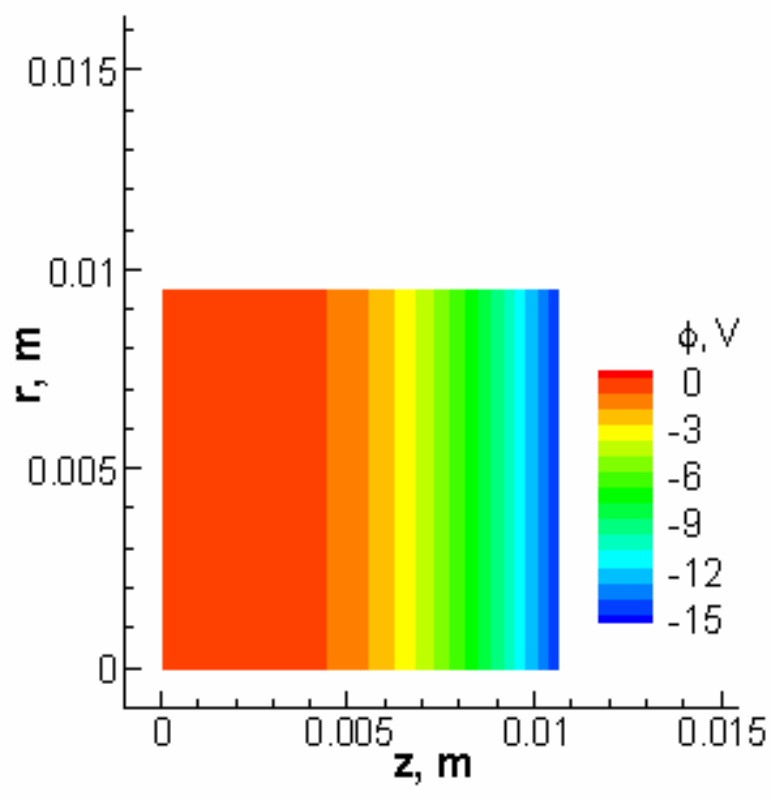

Figure 6. Simulated potential contours on the flux tube geometry for cold ions $\left(T_{i}=300 \mathrm{~K}\right)$ entering at the Bohm velocity $\left(v_{i s}=v_{B}=855 \mathrm{~m} / \mathrm{s}\right)$.



Figure 8. Simulated electron density contours for cold ions entering at $v_{B}$.

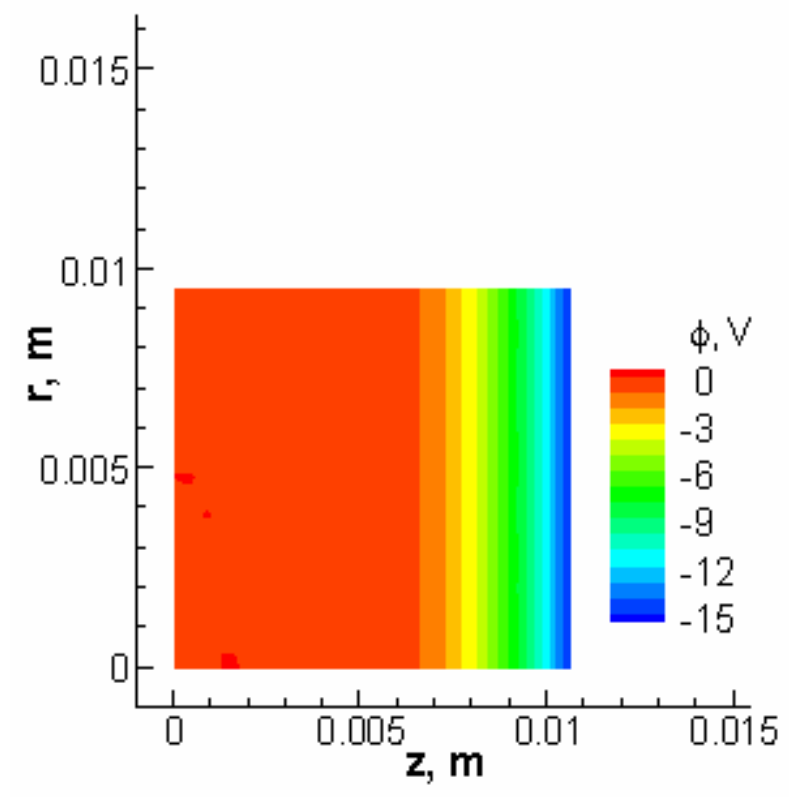

Figure 7. Simulated potential contours on the flux tube geometry for hot ions $\left(T_{i}=1 \mathrm{eV}\right)$ entering at the plume velocity $\left(v_{\text {is }}=v_{\text {Plume }}=2380 \mathrm{~m} / \mathrm{s}\right)$.

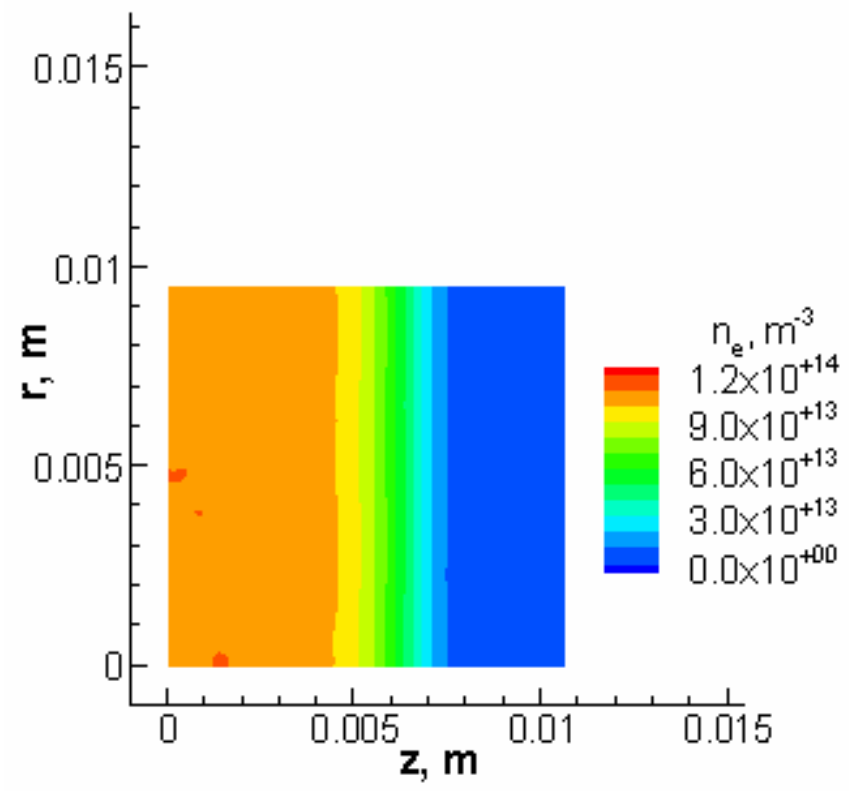

Figure 9. Simulated electron density contours for hot ions entering at $v_{\text {Plume }}$. 




Figure 10. Simulated ion density contours for cold ions entering at $v_{B}$.

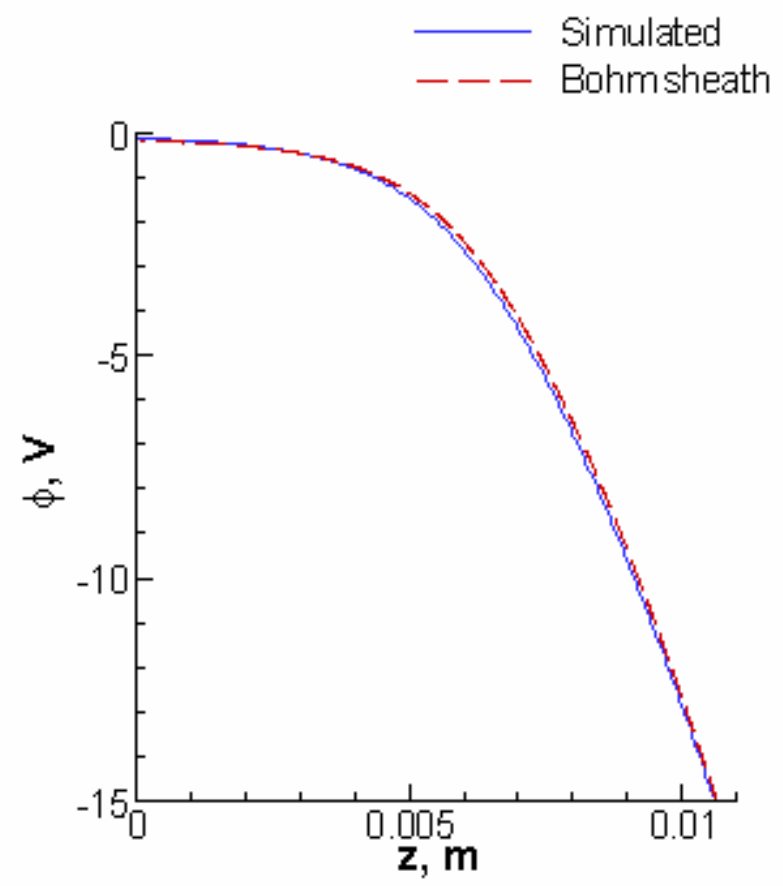

Figure 12. For cold ions entering at $v_{B}$, the radialaveraged plasma potential and Bohm sheath profile are in good agreement.

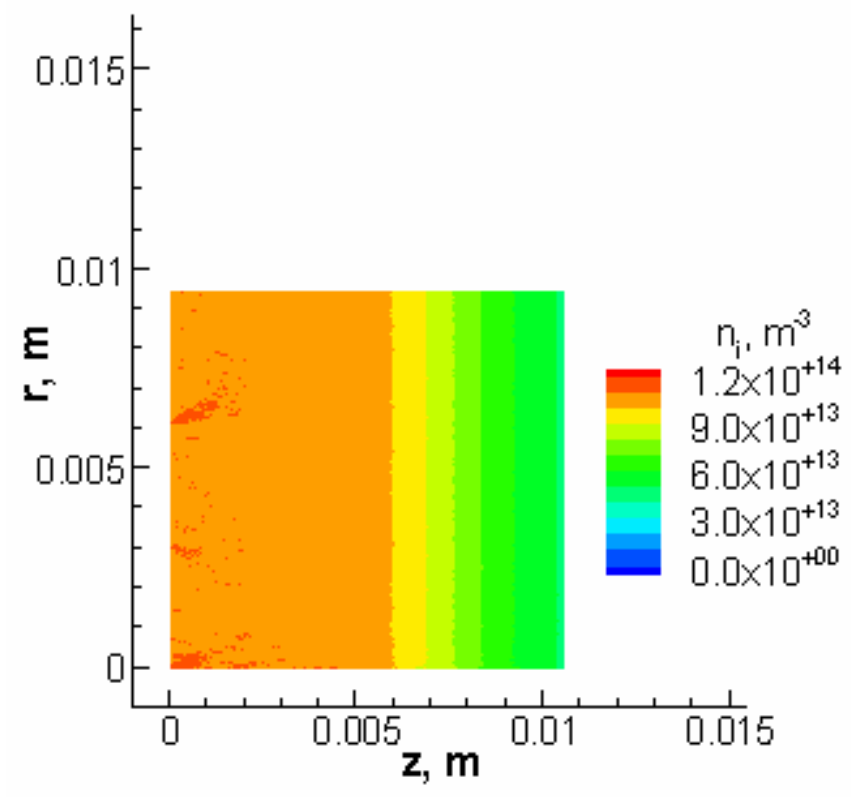

Figure 11. Simulated ion density contours for hot ions entering at $v_{\text {Plume }}$.



Figure 13. Likewise for hot ions entering at $v_{\text {Plume, }}$ the radial-averaged simulated plasma potential agrees with the Bohm sheath profile. 


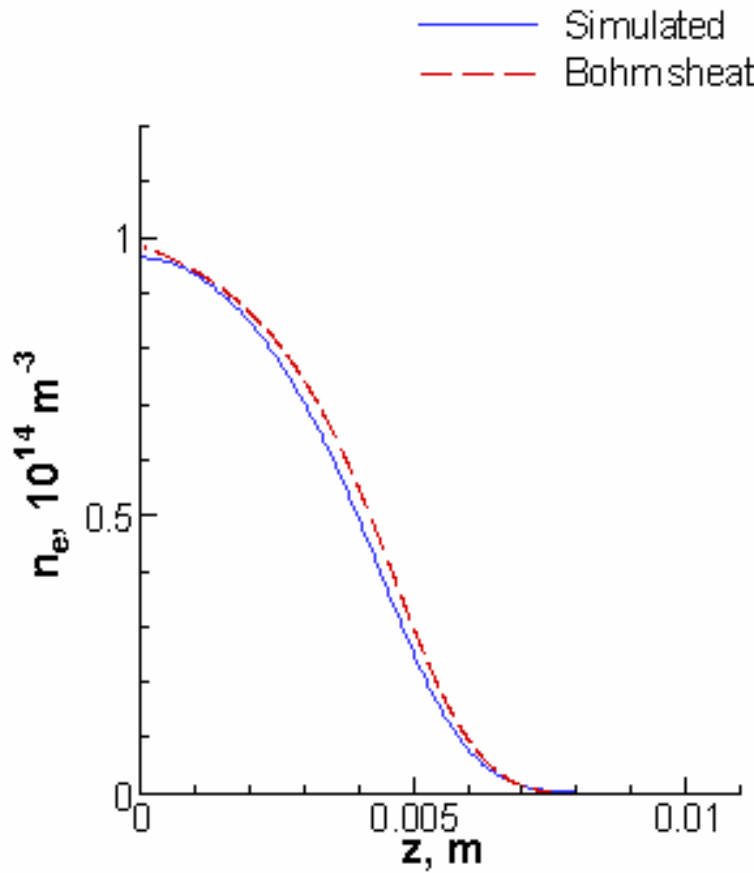

Figure 14. Bohm sheath and radial-average simulated electron densities for cold ions entering at $v_{B}$.

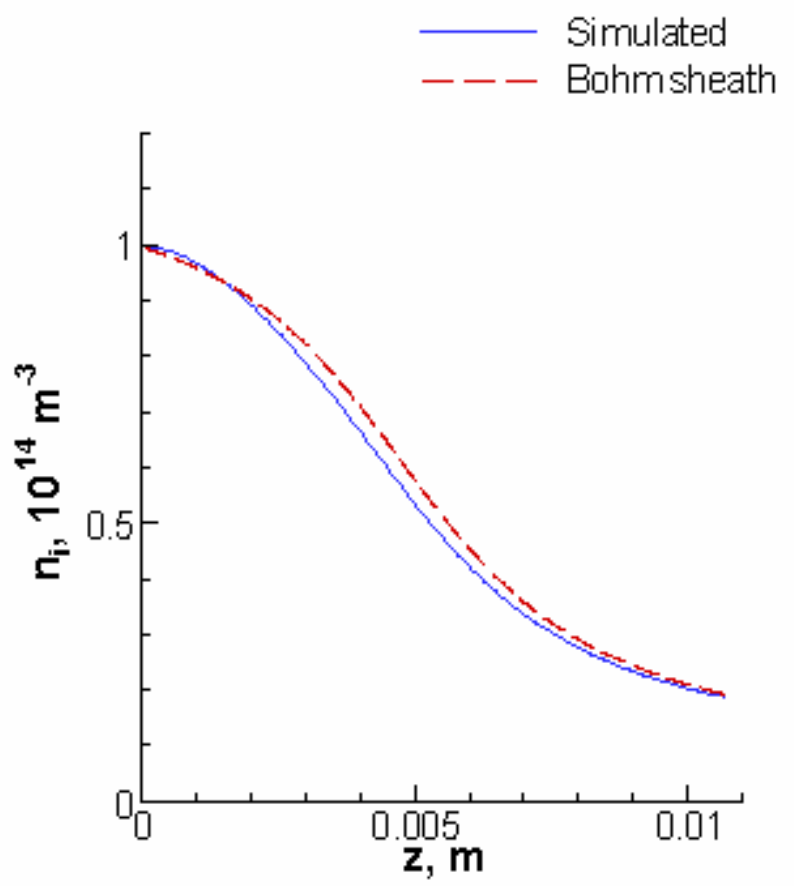

Figure 16. Bohm sheath and radial-average simulated ion densities for cold ions entering at $v_{B}$.

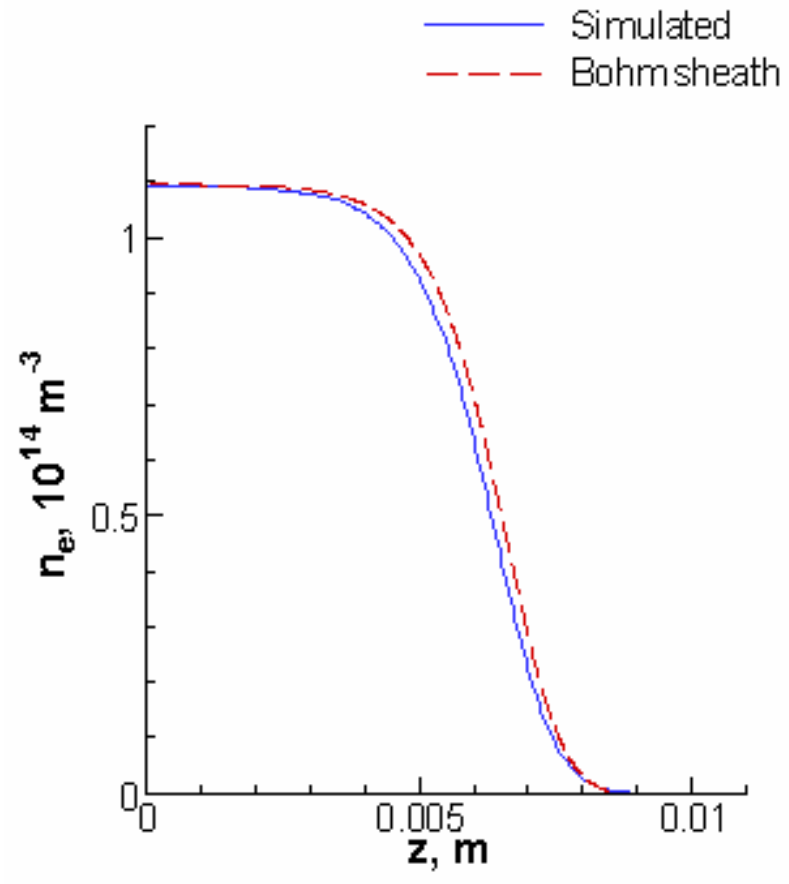

Figure 15. Bohm sheath and radial-average simulated electron densities for hot ions entering at $v_{\text {Plume. }}$



Figure 17. Bohm sheath and radial-average simulated ion densities for hot ions entering at $v_{\text {Plume }}$. 


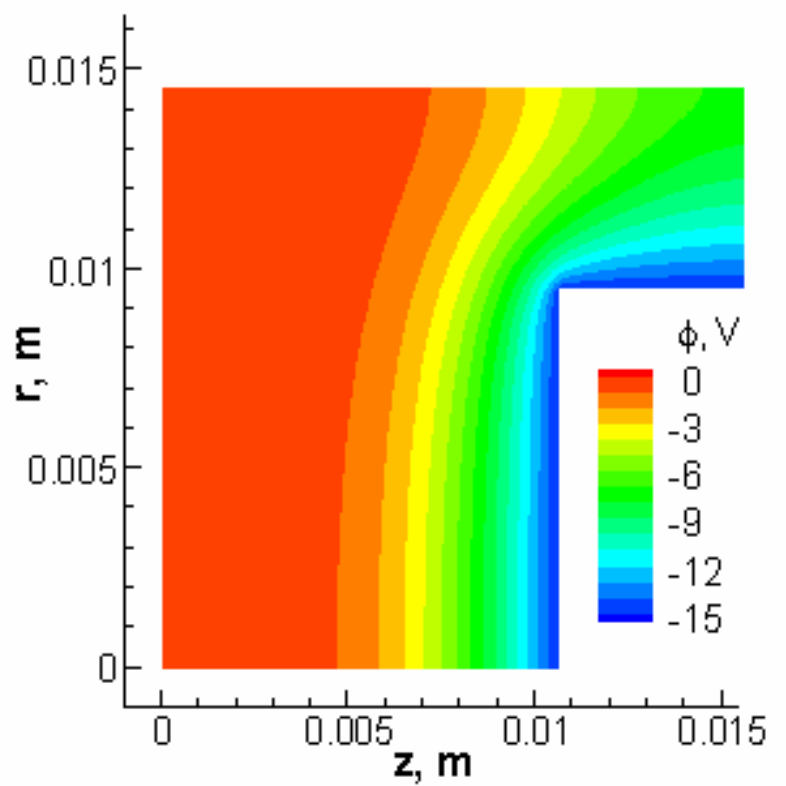

Figure 18. Simulated potential contours on the probe geometry for cold ions $\left(T_{i}=300 \mathrm{~K}\right)$ entering at the Bohm velocity $\left(v_{i s}=v_{B}=855 \mathrm{~m} / \mathrm{s}\right)$.



Figure 20. Simulated electron density contours for cold ions entering at $v_{B}$.



Figure 19. Simulated potential contours on the probe geometry for hot ions $\left(T_{i}=1 \mathrm{eV}\right)$ entering at the plume velocity $\left(v_{\text {is }}=v_{\text {Plume }}=2380 \mathrm{~m} / \mathrm{s}\right)$.

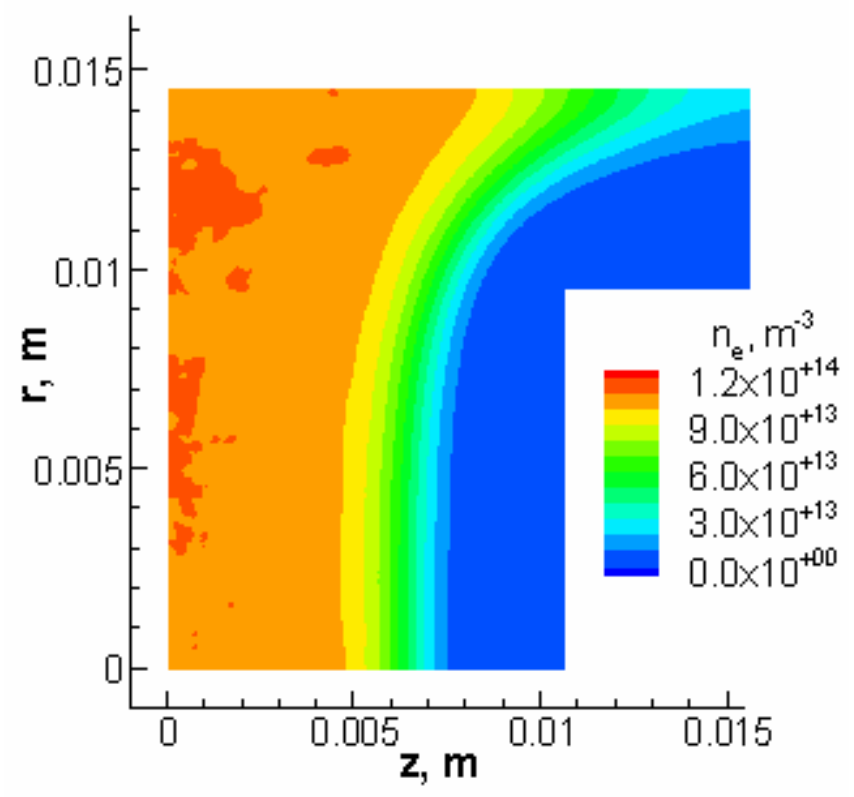

Figure 21. Simulated electron density contours for hot ions entering at $v_{\text {Plume. }}$ 




Figure 22. Simulated ion density contours for cold ions entering at $v_{B}$.



Figure 24. For cold ions entering at $v_{B}$, the radialaverage of plasma potential over the first $\mathbf{1 0 0}$ cells is slightly higher than the Bohm sheath profile.

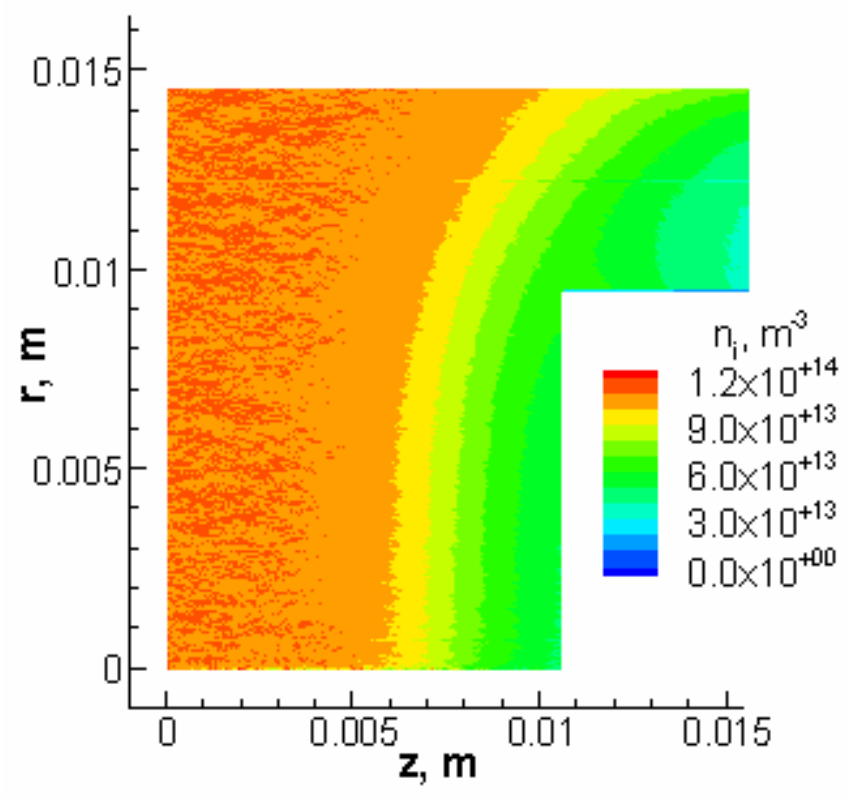

Figure 23. Simulated ion density contours for hot ions entering at $v_{\text {Plume }}$.

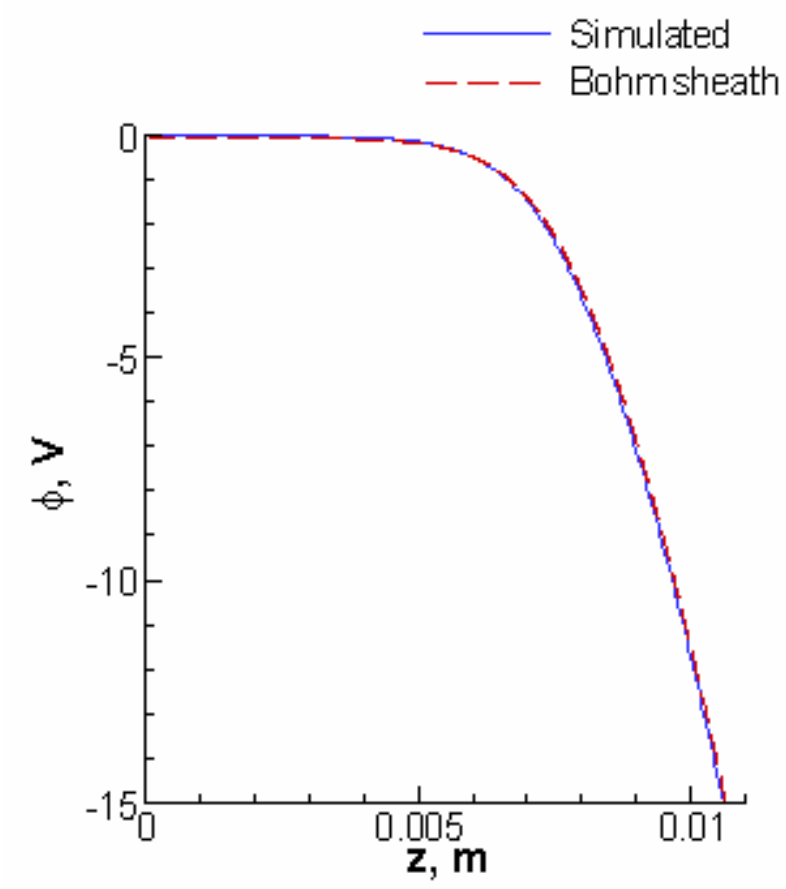

Figure 25. For hot ions entering at $v_{\text {Plume }}$, the limited radial-average of simulated plasma potential agrees with the Bohm sheath profile. 




Figure 26. Bohm sheath and radial-average simulated electron densities for cold ions entering at $v_{B}$.

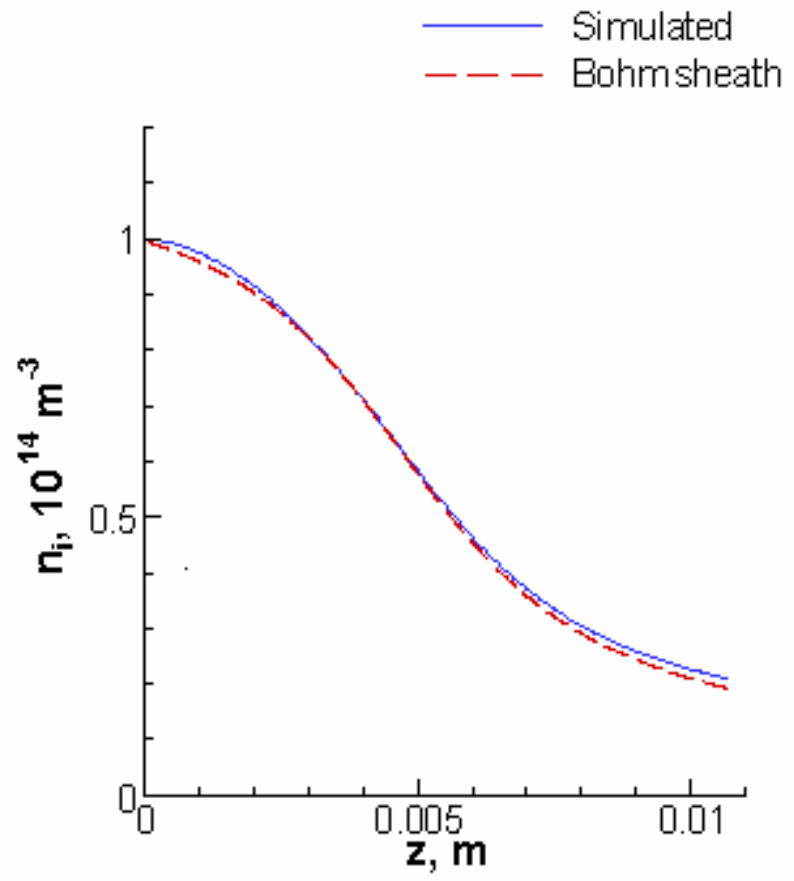

Figure 28. Bohm sheath and radial-average simulated ion densities for cold ions entering at $v_{B}$.

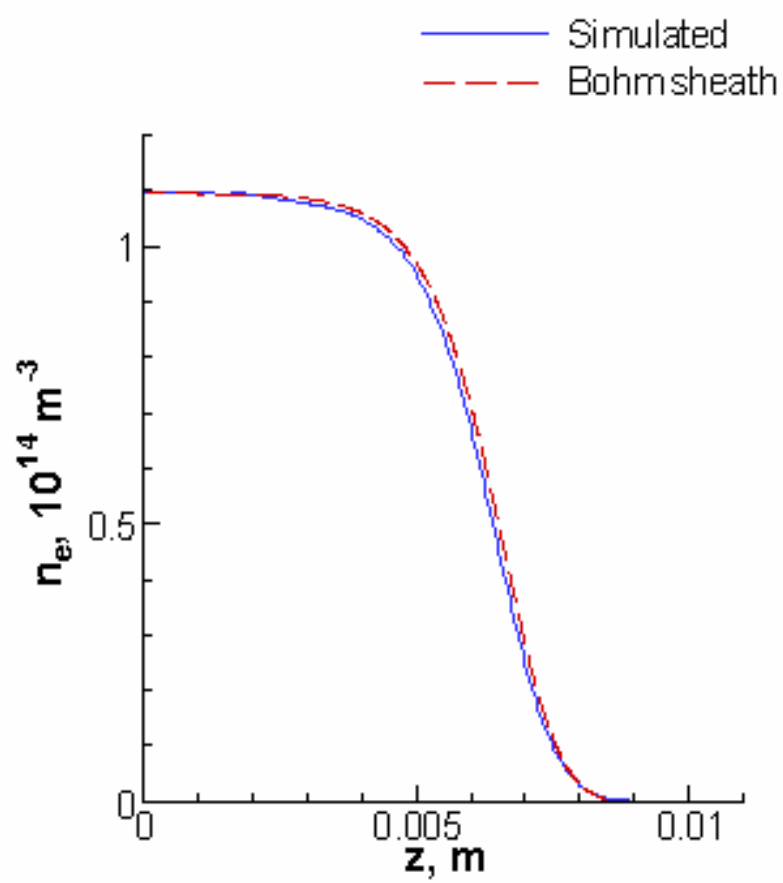

Figure 27. Bohm sheath and radial-average simulated electron densities for hot ions entering at $v_{\text {Plume }}$.

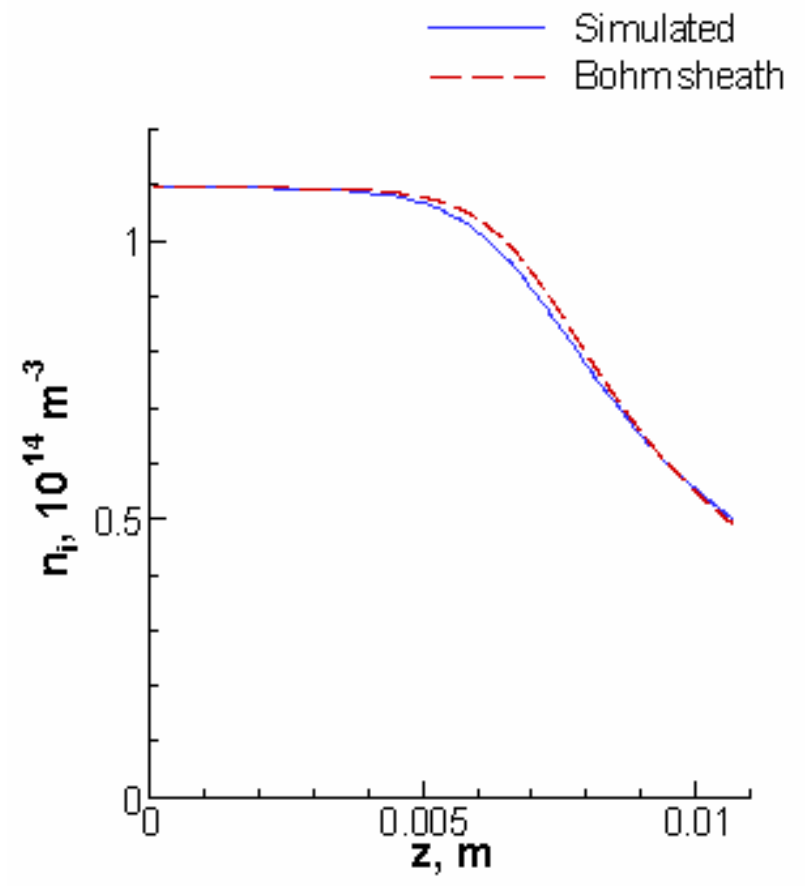

Figure 29. Bohm sheath and radial-average simulated ion densities for hot ions entering at $v_{\text {Plume }}$. 